\title{
ON THE STRENGTH OF MECHANICAL AND THERMAL DAMPING IN LINEAR MATERIALS*
}

\author{
BY \\ J. BIELAK ${ }^{1}$ AND R. C. MACCAMY ${ }^{2}$ \\ Carnegie Mellon University
}

\begin{abstract}
This paper gives a criterion for distinguishing between various types of linear models for elastic and thermoelastic behavior. The models are all dissipative and the steady-state periodic limit for periodic input can be defined. Associated with this limit is a scalar function of frequency which could be determined experimentally. Its behavior for large frequency determines the strength of the damping. Both fibers and one-dimensional bars are considered. The models include Kelvin-Voigt materials, viscoelastic models with regular and singular kernels as well as standard thermoelasticity. It is found that strength of damping increases the order of singularity in the viscoelastic models. Thermoelastic damping is shown to be weak and to be of the same order as for elastic models with smooth kernels.
\end{abstract}

1. Introduction. The purpose of this paper is the study of a class of linear, dissipative models for the elastic behavior of solids with emphasis on what we term strength of damping. We study both mechanical and thermal damping. The former can be discussed within the framework of fibers, while for the latter we need to consider the one-dimensional motion of bars.

We consider several models of mechanical damping. The simplest example of mechanical damping is a Kelvin-Voigt material $(\mathrm{K})$ which has a viscous effect. Our other mechanical models are all characterized by having the stress $\sigma$ as a linear functional $\mathscr{F}$ of the time history of strain $\varepsilon$. They are integral models. In this category is classical viscoelasticity $(\mathrm{V})$ with smooth kernels. We also consider two classes of models with singular kernels. The first $(\mathrm{R}-\mathrm{H})_{\alpha}, 0<\alpha<2$ was developed by Renardy and Hrusa, [5] and [7], and involves kernels which have singularities of order $-\alpha$. The second $(\mathrm{V}-\mathrm{K})_{\alpha}, 0<\alpha<1$ was introduced in [3] and also has singularities of order $-\alpha$.

Formally one has $(\mathrm{R}-\mathrm{H})_{\alpha} \rightarrow(\mathrm{V})$ and $(\mathrm{V}-\mathrm{K})_{\alpha} \rightarrow(\mathrm{V})$ as $\alpha \downarrow 0$ while $(\mathrm{V}-\mathrm{K})_{\alpha} \rightarrow(\mathrm{K})$ as $\alpha \uparrow 1$. ( $\mathrm{R}-\mathrm{H})_{\alpha}$ for $1<\alpha<2$ is similar to $(\mathrm{V}-\mathrm{K})_{\alpha}$ for $0<\alpha<1$; and we will not consider it in detail here. We note that the limits of $(\mathrm{R}-\mathrm{H})_{\alpha}$ as $\alpha \uparrow 2$ and $(\mathrm{V}-\mathrm{K})_{\alpha}$ as $\alpha \downarrow 0$ are not fully understood.

*Received January 13, 1989.

${ }^{1}$ This author was partially supported by the National Science Foundation under Grant ECE-8611060.

${ }^{2}$ This author was supported by the National Science Foundation under Grant DMS 8601288.

(C) 1989 Brown University 
References [5] and [7] contain a discussion of two concepts, propagation speed and smoothing of singularities for $(\mathrm{R}-\mathrm{H})_{\kappa}$. $(\mathrm{K})$ has infinite propagation speed and smooth singularities. (V) has finite propagation speed but does not smooth. For $\alpha \geq 1(\mathrm{R}-\mathrm{H})_{\alpha}$ is like $(\mathrm{K})$ but for $0<\alpha<1(\mathrm{R}-\mathrm{H})_{\alpha}$ has finite propagation speed and smooths. The models $(\mathrm{V}-\mathrm{H})_{\alpha}$ are like $(\mathrm{K})$ in this regard. If we think of smoothing as corresponding to stronger damping it follows that $(\mathrm{K}),(\mathrm{R}-\mathrm{H})_{\alpha}$ and $(\mathrm{V}-\mathrm{K})_{\alpha}$ are all stronger than $(\mathrm{V})$. The propagation speed concept seems to indicate that $(\mathrm{V}-\mathrm{K})_{\alpha}$, $0<\alpha<1$ and $(\mathrm{R}-\mathrm{H})_{\alpha}, 1 \leq \alpha<2$ are in some sense stronger than $(\mathrm{R}-\mathrm{H})_{\alpha}, 0<\alpha<1$.

In this paper we consider a different characterization of damping. This is somewhat more refined and distinguishes between the powers of $\alpha$ in the models. Moreover, for mechanical effects, it applies to the motion of fibers. It is suggested by observations in the survey article of Bert [1]. The idea is this. If a dissipative model has input data which tend to a time periodic limit of frequency $\omega$ the solution will have a similar limit. It will then be possible to define a steady-state power loss. This is a scalar function $P(\omega)$ of frequency which measures the power that must be provided per cycle to maintain the periodic motion and which can be determined experimentally.

It was observed in [1] that whereas for a Kelvin-Voigt material $P(\omega)$ is proportional to $\omega$, the power for many real materials exhibits a different dependene on $\omega$. For the materials considered here we find that for higher frequency, $P(\omega)$ can be of $O\left(\omega^{\prime \alpha}\right)$ for different $\alpha$ 's and, in fact, can be independent of $\omega$.

In Sec. 2 we make the above ideas precise for fibers. We find the following estimates for $P(\omega)$ when $\omega$ is large:

$$
\begin{gathered}
P(\omega)=O(\omega) \text { for }(\mathrm{K}), \quad P(\omega)=O\left(\omega^{-1}\right) \text { for }(\mathrm{V}) \\
P(\omega)=O\left(\omega^{\alpha-1}\right) \text { for }(\mathrm{R}-\mathrm{H})_{\alpha}, 0<\alpha \leq 1 \\
P(\omega)=O\left(\omega^{\alpha}\right) \text { for }(\mathrm{V}-\mathrm{K})_{\alpha}, 0<\alpha \leq 1 .
\end{gathered}
$$

We take the exponent of $\omega$ in the limit behavior of $P(\omega)$ as a measure of damping, larger exponent meaning stronger damping. (1.1) shows that we can get all exponents between -1 and +1 and in particular exponent zero for $(\mathrm{R}-\mathrm{H})_{1}$.

To study thermal effects we need to consider one-dimensional problems. In Sec. 3 we consider this first for mechanical damping. Then in Sec. 4 we consider a model in which the only damping is thermal $(\mathrm{T})$. The one-dimensional results are as follows for $\omega$ large:

$$
\begin{gathered}
P(\omega)=O\left(\omega^{3 / 2}\right) \quad \text { for }(\mathrm{K}), \quad P(\omega)=O(\omega) \text { for }(\mathrm{V}) \\
P(\omega)=O\left(\omega^{1+\alpha / 2}\right) \text { for }(\mathrm{V}-\mathrm{K})_{\alpha}, 0<\alpha<1 \\
P(\omega) \sim c_{\alpha} \omega, \quad c_{\alpha} \rightarrow \infty \text { as } \alpha \uparrow 1 \text { for }(\mathrm{R}-\mathrm{H})_{\alpha}, 0<\alpha<1 \\
P(\omega)=O(\omega) \text { for }(\mathrm{T}) .
\end{gathered}
$$

We note that the range of exponents for $\omega$ is less for the bars than the fibers. In particular the exponent above no longer distinguishes between the $(\mathrm{R}-\mathrm{H})_{\alpha}$ models, all giving the same exponent. The constant $c_{\alpha}$ is needed for a more refined estimate. Note also that the exponent for $(\mathrm{T})$ is the same as that for $(\mathrm{V})$ and we conclude that 
thermoelastic damping is weak. We will see in the last section that for a thermally damped system one can find a viscoelastic system with exactly the same asymptotic behavior for $P(\omega)$.

2. Mechanical damping in fibers. Suppose a unit mass is attached to an elastic fiber and is subjected to a force $f$. The equation of motion of the mass is

$$
D^{2} \varepsilon=-\sigma+f
$$

where $D$ denotes time differentiation, $\varepsilon$ is the strain and $\sigma$ the stress. From $(2.1)$ we have

$$
f D \varepsilon=D\left(\frac{1}{2}(D \varepsilon)^{2}\right)+\sigma D \varepsilon \equiv p[\varepsilon] .
$$

The left side of (2.2) gives the power that must be applied by the force $f$ in order to maintain the motion, and could be measured experimentally.

One obtains models by giving constitutive assumptions relating $\sigma$ and $\varepsilon$. The class we want to consider is to be dissipative. This means that such models have an internal damping mechanism so that, roughly, if $f(t) \rightarrow 0$ as $t \rightarrow \infty$ so does $\varepsilon(t)$. Related to this is approach to steady-state. More precisely the property we want is the following. Suppose

$$
f(t)=\operatorname{Re}\left(f_{0} e^{i \omega t}\right)+F(t)
$$

where $F(t) \rightarrow 0$ as $t \rightarrow 0$ in a sense to be made precise. Then we want to have an $\varepsilon_{0}(\omega)$ such that

$$
\varepsilon(t)=\operatorname{Re}\left(\varepsilon_{0} e^{i \omega t}\right)+\varepsilon_{1}(t), \quad \varepsilon_{1}(t) \rightarrow 0 \quad \text { as } t \rightarrow \infty .
$$

In these situations it will also be true that we will have, ${ }^{3}$

$$
\int_{t}^{t+2 \pi / \omega} p[\varepsilon](\tau) d \tau \rightarrow P(\omega)\left|\varepsilon_{0}\right|^{2} \quad \text { as } t \rightarrow \infty .
$$

We term $P(\omega)$ the steady-state power loss over a cycle. We will show that the asymptotic behavior of $P(\omega)$ for large $\omega$ enables us to distinguish between types of models. We consider this behavior as a measure of the strength of dissipation, larger $P(\omega)$ meaning greater damping. We observe that in all cases $P(\omega)=O(\omega)$ for small $\omega$; hence this behavior does not distinguish models.

The simplest model is Hooke's law $\sigma=E \varepsilon$ but this is not dissipative. Our models all have the following form. Let $\varepsilon^{t}$ denote the strain history, $\varepsilon^{t}(\tau)=\varepsilon(t-\tau)$. Then we assume there is a linear functional $\mathscr{F}$ such that

$$
\sigma(t)=\mathscr{F}\left[\varepsilon^{t}\right] .
$$

With the assumption (2.4), (2.2) requires the initial history $\varepsilon(t), t \leq 0$ and we assume throughout that this is zero.

\footnotetext{
${ }^{3}$ If this were to be done experimentally, one would adjust the force amplitude to keep the displacement amplitude constant.
} 
Let us describe the $\mathscr{F}$ 's for the models in the introduction. The first two are the familiar Kelvin-Voigt and viscoelasticity:

$$
\begin{gathered}
\mathscr{F}\left[\varepsilon^{t}\right]=E \varepsilon(t)+\lambda D \varepsilon(t), \quad E>0, \lambda>0, \\
\mathscr{F}\left[\varepsilon^{t}\right]=a(0) \varepsilon(t)+\int_{0}^{\infty} D a(\tau) \varepsilon(t-\tau) d \tau,
\end{gathered}
$$

where $a$ is a smooth kernel. The other models involve singular kernels. For $0<\alpha<2$ put $\gamma_{\alpha}(\tau)=\tau^{-\alpha}$. Then we have,

$$
\begin{gathered}
\mathscr{F}\left[\varepsilon^{t}\right]=M \varepsilon(t)+\int_{0}^{\infty} m_{\alpha}(\tau)(\varepsilon(t)-\varepsilon(t-\tau)) d \tau, \quad M>0 \\
0<\alpha<2, m_{\alpha}(\tau)=\gamma_{\alpha}(\tau) m(\tau), m \text { regular at } 0, \\
\mathscr{F}\left[\varepsilon^{\tau}\right]=M \varepsilon(t)+D \int_{0}^{\infty} b_{\alpha}(\tau) \varepsilon(t-\tau) d \tau, \quad M>0 \\
0<\alpha<1, b_{\kappa}(\tau)=(\Gamma(1-\alpha))^{-1} \gamma_{\alpha}(\tau) b(\tau), b \text { regular at } 0,
\end{gathered}
$$

where $\Gamma$ is the gamma function.

REMARKS 2.1 .

(i) For $\alpha \geq 1, m_{\alpha}$ is not integrable. However, when $\tau$ is small we have $\varepsilon(t)-$ $\varepsilon(t-\tau) \sim-D \varepsilon(t) \tau$ so that $(\mathrm{R}-\mathrm{H})_{\alpha}$ is meaningful for $1 \leq \alpha<2$. For $0<\alpha<1$ we assume $m_{c} \in L_{1}(0, \infty)$ and then $(\mathrm{R}-\mathrm{H})_{\alpha}$ can be written

$$
\begin{array}{r}
\mathscr{F}\left[\varepsilon^{t}\right]=\left(M+\hat{m}_{\alpha}(0)\right) \varepsilon(t)-\int_{0}^{\infty} m_{\alpha}(\tau) \varepsilon(t-\tau) d \tau \\
0<\alpha<1, \hat{m}_{\alpha}(0)=\int_{0}^{\infty} m_{\alpha}(\tau) d \tau .
\end{array}
$$

This is the only case we consider here except for a remark at the end of the section.

(ii) We see that, formally, both $(\mathrm{R}-\mathrm{H})_{\alpha}$ and $(\mathrm{V}-\mathrm{K})_{\alpha}$ tend to $(\mathrm{V})$ as $\alpha \downarrow 0$ for an appropriate $a$. We have, because of the gamma function,

$$
\lim _{\kappa \uparrow 1} \int_{0}^{\infty} b_{r(}(\tau) \varepsilon(t-\tau) d \tau=b(0) \varepsilon(t)
$$

so that, formally $(\mathrm{V}-\mathrm{K})_{x}$ tends to $(\mathrm{K})$ for appropriate $E$ and $\lambda$. On the other hand the limit of $(\mathrm{R}-\mathrm{H})_{\alpha}$ as $\alpha \uparrow 1$ is complicated. (See Remark (2.2).)

Before we give precise statements of results let us motivate them. We treat $(2.1)$ with $f$ as in (2.3) by solving the complex problem,

$$
D^{2} \mathscr{E}(t)=-\mathscr{F}\left[\mathscr{E}^{t}\right]+f_{0} \varepsilon^{i \omega t}+F(t)
$$

and then taking $\varepsilon(t)=\operatorname{Re} \mathscr{E}(t)$. For each of our functionals there will be a Laplace transform $\hat{\mathscr{F}}(s)$ such that (for $\varepsilon(t) \equiv 0$ in $t<0$ )

$$
\hat{\sigma}(s)=\mathscr{L}[\sigma(\cdot)](s)=\hat{\mathscr{F}}(s) \mathscr{L}\left[\varepsilon^{\cdot}\right](s)=\hat{\mathscr{F}}(s) \hat{\varepsilon}(s) .
$$

Transforming (2.5) yields,

$$
\hat{\mathscr{E}}(s)=\left(s^{2}+\hat{\mathscr{F}}(s)\right)^{-1}\left(f_{0}(s-i \omega)^{-1}+\hat{F}(s)\right) .
$$

We can then try to recover $\mathscr{E}(t)$ by the formula,

$$
\mathscr{E}(t)=(2 \pi i)^{-1} \int_{c-i \infty}^{c+i \infty} e^{s t} \hat{\mathscr{E}}(s) d s .
$$


We want to use $(2.8)$ for $c=0$. This motivates the following definitions:

DEFINITION.

(i) $\mathscr{L}=$ set of all function $\hat{\mathscr{F}}(s)$ which are continuous in $\operatorname{Re} s \geq 0$ and analytic in $\operatorname{Re} s>0$.

(ii) $\mathscr{F}$ is a dissipative functional if $\hat{\mathscr{F}} \in \mathscr{L}$ and

$$
s^{2}+\hat{\mathscr{F}}(s) \neq 0 \text { in } \operatorname{Re} s \geq 0 .
$$

Suppose we have $\hat{F} \in \mathscr{L}$ and $\mathscr{F}$ is dissipative; then from $(2.7) \hat{\mathscr{E}}(s)$ will be regular in $\operatorname{Re} s \geq 0$ except for a simple pole at $s=i \omega$ and this, with (2.8), will produce (I).

Our first condition guarantees that $\hat{F} \in \mathscr{L}$ and is:

$$
D^{j} F \in C[0, \infty) \cap L^{1}(0, \infty), \quad j=0,1,2 ; \quad F(0)=0 .^{4}
$$

Note that $(\mathrm{F})$ implies $D^{j} F(t) \rightarrow 0$ as $t \rightarrow \infty$ for $j=0,1$.

Let us suppose that we know that $\hat{F} \in \mathscr{L}$. Then we can give a simple condition which guarantees (2.9).

Proposition 2.1. If $\hat{\mathscr{F}} \in \mathscr{L}$ then $\mathscr{F}$ is dissipative if

$$
\hat{\mathscr{F}}(0) \neq 0 \text { and } \operatorname{Re}(\hat{\mathscr{F}}(i \eta) / i \eta) \neq 0 \text { for } \eta \neq 0 \text {. }
$$

Proof. The first condition guarantees that $s^{2}+\hat{\mathscr{F}}(s)$ is nonzero for $s$ small. We write $s^{2}+\mathscr{F}(s)=s \hat{\chi}(s), \chi(s)=(s+\hat{F}(s) / s)$. The second condition guarantees that $\chi(i \eta) \neq 0$ and then, by the maximum principle $\chi(s) \neq 0$ in $\operatorname{Re} s \geq 0, s \neq 0$.

Let us list the $\hat{\mathscr{F}}$ 's for our models:

$$
\begin{aligned}
& \hat{\mathscr{F}}(s)=E+\lambda s \text { for }(\mathrm{K}) \\
& \hat{\mathscr{F}}(s)=s \hat{a}(s) \text { for }(\mathrm{V}) \\
& \hat{\mathscr{F}}(s)=M+\hat{m}_{\alpha}(0)-\hat{m}_{\alpha}(s), \quad 0<\alpha<1 \quad \text { for }(\mathrm{R}-\mathrm{H})_{\alpha} \\
& \hat{\mathscr{F}}(s)=M+s \hat{b}_{\alpha}(s) \quad \text { for }(\mathrm{V}-\mathrm{K})_{\alpha} .
\end{aligned}
$$

It is clear that $\mathscr{F}$ is dissipative for $(\mathrm{K})$ but for the others we need some hypotheses. We will also need the behavior of $\hat{\mathscr{F}}(s)$ for large $s$. We list the hypotheses we require. For any $k$ we write $k^{1}$ for the function $t \rightarrow t k(t)$. For $(\mathrm{V})$ :

$$
\begin{gathered}
a \in C^{(3)}[0, \infty), \quad a(0)>0, \quad D a(0)<0 \\
a(t)=a_{\infty}+A(t), \quad a_{\infty}>0, \quad D^{j} A \in L_{1}(0, \infty), \quad j \leq 3, \quad A^{1} \in L^{1}(0, \infty) \\
\operatorname{Re} \hat{A}(\text { in) }>0, \quad-\infty<\eta<\infty .
\end{gathered}
$$

For $(\mathrm{R}-\mathrm{H})_{\alpha}$ :

$$
\begin{gathered}
m_{\alpha} \quad \text { and } \quad m_{\alpha}^{1} \in L_{1}(0, \infty) ; \quad \operatorname{sgn} \eta \operatorname{Im} \hat{m}_{\alpha}(i \eta)<0 \quad \text { for } n \neq 0 \\
\hat{m}_{\alpha}(s)=\left(\Gamma(1-\alpha) A_{\alpha} s^{\alpha-1}+O\left(s^{\alpha-2}\right) \quad \text { as } s \rightarrow \infty .\right.
\end{gathered}
$$

For $(\mathrm{V}-\mathrm{K})$ :

$$
\begin{aligned}
b_{\alpha} \quad \text { and } \quad b_{\alpha}^{1} & \in L_{1}(0, \infty), \quad \operatorname{Re} \hat{b}(i \eta)>0 \text { for } \eta \neq 0 \\
\hat{b}_{\alpha}(s) & =A_{\alpha} s^{\alpha-1}+O\left(s^{\alpha-2}\right) \quad \text { as } s \rightarrow \infty .
\end{aligned}
$$

Remark 2.3 contains some observations about these conditions. We have the following results when (2.3), (F) and these conditions hold.

${ }^{4}$ See remark A.1 in the appendix. 
LEMMA 2.1. The functionals $\mathscr{F}$ are all dissipative.

THEOREM 2.1. There exists a unique solution of (2.1) which satisfies (I) with,

$$
\varepsilon_{0}(\omega)=\left(-\omega^{2}+\hat{\mathscr{F}}(i \omega)\right) f_{0} .
$$

THEOREM 2.2. Condition (II) is satisfied with,

$$
P(\omega)=\pi \operatorname{Im} \hat{\mathscr{F}}(i \omega) .
$$

Theorem 2.3. For large $\omega$ we have

$$
\begin{gathered}
P(\omega) \sim \pi \lambda \omega \quad \text { for }(\mathrm{K}), \quad P(\omega) \sim-\pi D a(0) \omega^{-1} \text { for }(\mathrm{V}) \\
P(\omega) \sim\left(-\pi \Gamma(1-\alpha) A_{\alpha} \sin \frac{\pi}{2}(1-\alpha)\right) \omega^{\alpha-1} \text { for }(\mathrm{R}-\mathrm{H})_{\alpha} \\
P(\omega) \sim\left(\pi A_{\alpha} \sin \frac{\pi \alpha}{2}\right) \omega^{\alpha} \text { for }(\mathrm{V}-\mathrm{K})_{\alpha} .
\end{gathered}
$$

Proof of Lemma 2.1. We see that our conditions guarantee that $\hat{F} \in \mathscr{L}^{5}$ and then Lemma 2.1 follows from Proposition 2.1.

The complete proof of Theorem 2.1 is a little technical and we give it in the appendix. We sketch the proof of Theorem 2.2. We write for the complex solution of $(2.5), \mathscr{E}(t)=\varepsilon_{0} e^{i \omega t}+\mathscr{E}_{1}(t)$. We will see in the proof of Theroem 2.1 that $\mathscr{E}_{1}(t)$ and $D \mathscr{E}_{1}(t) \rightarrow 0$ as $t \rightarrow \infty$ and then it can be verified that as $t \rightarrow \infty$,

$$
\mathscr{F}\left(\mathscr{C}^{t}\right) \sim \varepsilon_{0} \hat{\mathscr{F}}(i \omega) e^{i \omega t} \equiv \sigma_{0} e^{i \omega t} .
$$

It follows that the limit of the integral in (II) is obtained by replacing $\varepsilon$ by $\operatorname{Re} \varepsilon_{0} e^{i \omega t}$ in the right side of (2.2) and integrating. This yields,

$$
\begin{aligned}
\int_{t}^{t+2 \pi / \omega} p[\varepsilon](\tau) d \tau & \sim \int_{t}^{t+2 \pi / \omega}\left(\operatorname{Re} \sigma_{0} e^{i \omega \tau}\right) D \operatorname{Re}\left(\varepsilon_{0} e^{i \omega \tau}\right) d \tau \\
& =\frac{1}{2} \operatorname{Re} \int_{t}^{t+2 \pi / \omega} \sigma_{0}(-i \omega) \varepsilon_{0} d \tau=\pi \operatorname{Re}(-i \hat{\mathscr{F}}(i \omega))\left|\varepsilon_{0}\right|^{2}
\end{aligned}
$$

which is $(2.12)$. The estimates $(2.13)$ then follow from $(2.12),(2.10),\left(C_{R N}\right),\left(C_{V K}\right)$ and the following consequence of $\left(\mathrm{C}_{\mathrm{V}}\right)$ :

$$
s \hat{a}(s)=a(0)+D a(0) s^{-1}+O\left(s^{-2}\right) .
$$

REMARK 2.2. Note that as $\alpha \rightarrow 1, \Gamma(1-\alpha) \sin \frac{\pi}{2}(1-\alpha) \rightarrow \pi / 2$ so that if $A_{\alpha}$ has a limit at $\alpha=1,(2.13)$ shows that in the limit $\alpha \uparrow 1, p(\omega)$ is independent of $\omega$ for $(\mathrm{R}-\mathrm{H})$. We are not sure about the limit as $\alpha \downarrow 0$ of $(\mathrm{V}-\mathrm{K})_{\alpha}$.

REMARK 2.3. Prototype kernels for $(\mathrm{R}-\mathrm{H})_{\alpha}$ and $(\mathrm{V}-\mathrm{K})_{\alpha}$ are,

$$
m_{\alpha}(t)=t^{-\alpha} e^{-\eta t}, \quad b_{\alpha}(t)=(\Gamma(1-\alpha))^{-1} t^{-\alpha} e^{-\eta t}, \quad \eta>0 .
$$

These yield,

$$
\begin{aligned}
& \hat{\mathscr{F}}(s)=M+\Gamma(1-\alpha)\left(\eta^{\alpha-1}-(s+\eta)^{\alpha-1}\right) \text { for }(\mathrm{R}-\mathrm{H})_{\alpha} \\
& \mathscr{F}(s)=M+s(s+\eta)^{\alpha-1} \text { for }(\mathrm{V}-\mathrm{K})_{\alpha}
\end{aligned}
$$

and conditions $\left(\mathrm{C}_{\mathrm{RH}}\right)$ and $\left(\mathrm{C}_{\mathrm{VK}}\right)$ can be verified directly.

$\overline{{ }^{5} \text { Note that for }(\mathrm{V}) \cdot \hat{F}} \in \mathscr{L}$ only if $a_{\infty} \neq 0$. 
REMARK 2.4. Biot [2] has proposed a model with the same frequency independent behavior at high frequencies as $(\mathrm{R}-\mathrm{H})_{1}$. In fact it can be shown that the Biot model is the same as $(\mathrm{R}-\mathrm{H})_{1}$ with $m_{1}$ given by $(2.16)$ and that $P(\omega)=\tan (\omega / \eta)$ in which $\eta$, the constant appearing in (2.16), is the relaxation frequency. Thus, $P(\omega)$ becomes practically independent of frequency even for small $\omega$, provided $\eta$ is small.

REMARK 2.5. The transform conditions on the kernels are familiar in the study of viscoelasticity see [5]. The following facts are known. Suppose $D^{j} k \in C[0, \infty) \cap$ $L^{1}(0, \infty)$. Then:

$$
\begin{gathered}
(-1)^{j} D^{j} k>0 \quad j=0,1,2 \Rightarrow \operatorname{Re} \hat{k}(i \eta)>0 \\
(-1)^{j} D^{j} k>0 \quad j=0,1 \Rightarrow \operatorname{sgn} \eta \operatorname{Im} \hat{k}(i \eta)<0 .
\end{gathered}
$$

However there are kernels $k$ which are oscillatory and still satisfy one of these conditions. For instance, if $k(t)=e^{-\mu t} \cos \gamma t, \operatorname{Re} \hat{k}(i \eta)>0$ and if $k(t)=e^{-\mu t} \sin \gamma t$ then $\operatorname{sgn} \eta \hat{k}(i \eta)<0$. This remark can be applied directly to yield sufficient conditions for $\left(C_{V}\right)$. It was observed in [3] that a class of kernels satisfying $\left(C_{V K}\right)$ can be generated by putting

$$
b_{r k}(t)=(\Gamma(1-\alpha))^{-1} \int_{0}^{t}(t-\tau)^{-\alpha} e^{-\beta(t-\tau)} k(t) d \tau
$$

where $\eta$ and $\gamma$ are positive and $D^{j} k \in C[0, \infty) \cap L^{1}(0, \infty)$ with $(-1)^{j} D^{j} k(t)>0$. One can show that the same formula with the same conditions on $k$ but without the factor $(\Gamma(1-\alpha))^{-1}$ will generate a class of kernels satisfying $\left(\mathrm{C}_{\mathrm{RH}}\right)$.

REMARK 2.6. We comment on ( $\mathrm{R}-\mathrm{H})_{c}^{\prime}$ for $\alpha \geq 1$. It is noted in [4] that the quantity $\hat{\mathscr{F}}(s)$ is still meaningful in this case but that it needs to be interpreted as $\hat{\mathscr{F}}(s)=M+\int_{0}^{\infty} m_{\alpha}(t)\left(1-e^{-s t}\right) d t$. If one again chooses $m_{\alpha}(t)=t^{-\alpha} e^{-\eta t}, \eta>0$ this quantity will behave like $s^{\alpha-1}$ as $s \rightarrow \infty$. This means that $P(\omega)$ will be of order $\omega^{\alpha-1}$ so that this model behaves the same way as $(\mathrm{V}-\mathrm{K})_{\alpha-1}$. However, the limit as $\alpha \uparrow 2$ will not exist.

3. Mechanical damping in bars. We consider one-dimensional longitudinal motion of a homogeneous bar of uniform cross section. Let $x$ be a position in the unstretched configuration and $u(x, t)$ be displacement with $0<x<L$. Assume the bar has unit density and there are no body forces. Then the equation of motion is,

$$
u_{t t}(x, t)=\sigma_{x}(x, t)
$$

where $\sigma$ is the stress.

We suppose the bar is initially at rest, is damped at $x=0$, and subject to a prescribed displacement $\varphi(t)$ at $x=L$. Thus

$$
\begin{gathered}
u(x, 0)=u_{t}(x, 0)=0, \quad 0<x<L, t \leq 0 \\
u(0, t)=0, \quad u(L, t)=\varphi(t), \quad t>0 .
\end{gathered}
$$

We use the same models for stress-strain relations as in Sec. 2 but in the present situation we have $\varepsilon(x, t)=u_{x}(x, t)$. There is an analog of the power loss idea here. 
We have, by (3.1),

$$
\begin{aligned}
\sigma(L, t) D \varphi(t) & =\sigma(L, t) u_{t}(L, t) \\
& =\int_{0}^{L} u_{t t}(x, t) u_{t}(x, t) d x+\int_{0}^{L} \sigma(x, t) \varepsilon_{t}(x, t) d x \\
& \equiv p[u](t) .
\end{aligned}
$$

Once again the left side is the power that must be applied and is again capable of measurement.

We again assume $\varphi$ has the form (2.3) $\left(\varphi(t)=\operatorname{Re}\left(\varphi_{0} e^{i \omega t}\right)+F(t)\right)$ and make the same assumptions about the functionals $\mathscr{F}$ as in Sec. 2 . Then we obtain analogous results. We define the function $\gamma(s)$ by

$$
\gamma(s)=\frac{s}{\sqrt{\hat{\mathscr{F}}(s)}}
$$

and the function $P(\omega)$ by,

$$
\int_{t}^{t+2 \pi / \omega} p[u](\tau) d \tau \rightarrow P(\omega)\left|\varphi_{0}\right|^{2} \quad \text { as } t \rightarrow \infty .
$$

THEOREM 3.1. There exists a unique solution $u$ of (3.1), (3.2) with,

$$
u(x, t)=\operatorname{Re}\left(u^{0}(x, \omega) e^{i \omega t}\right)+u^{1}(x, t)
$$

where $u^{1}(x, t) \rightarrow 0$ as $t \rightarrow \infty$ and

$$
u^{0}(x, \omega)=f_{0} \frac{\sinh \gamma(i \omega) x}{\sinh \gamma(i \omega) L} .
$$

Theorem 3.2. $P(\omega)$ exists and

$$
P(\omega)=\operatorname{Im}(i \omega \sqrt{\hat{\mathscr{F}}(i \omega)} \operatorname{ctnh} \gamma(i \omega) L)\left|\varphi_{0}\right|^{2} .
$$

TheOREM 3.3. As $\omega \rightarrow \infty$ we have,

$$
\begin{aligned}
& P(\omega)=O\left(\omega^{3 / 2}\right) \text { for }(\mathrm{K}) \\
& P(\omega)=O(\omega) \text { for }(\mathrm{V}) \\
& P(\omega)=O\left(\omega^{-1+\alpha / 2}\right) \text { for }(\mathrm{V}-\mathrm{K}) \\
& P(\omega) \sim\left(M+\Gamma(1-\alpha) m^{1-\alpha}\right) \omega \text { for }(\mathrm{R}-\mathrm{H}) .
\end{aligned}
$$

Note that the coefficient in (R-H) tends to infinity as $\alpha \uparrow 1$.

Once again some details of the proof are postponed until the appendix but we can describe the essential ideas which are the same as in Sec. 2.

We seek a complex solution $U$ for $\varphi=\varphi_{0} e^{i \omega t}+F$ and then take the real part. Transforming with respect to time gives

$$
\begin{gathered}
s^{2} \hat{U}(x, s)=\hat{\mathscr{F}}(s) \hat{U}_{x x}(x, s), \quad 0<x<L \\
\hat{U}(0, s)=0, \quad \hat{U}(L, s)=\hat{f}(s) .
\end{gathered}
$$

Then, by (3.4), we have,

$$
\hat{U}(x, s)=\hat{\varphi}(s)(\sinh \hat{\gamma}(s) x) / \sinh \gamma(s) L .
$$


Once again we recover $U$ from the formula,

$$
U(x, t)=(2 \pi i)^{-1} \int_{c-i \infty}^{c+i \infty} e^{s t} \hat{U}(x, s) d s .
$$

The analog of dissipativity here is the statement that $\sinh \gamma(s) L \neq 0$ in $\operatorname{Re} s \geq 0$. Thus if $\hat{f}$ is continuous in $\operatorname{Re} s \geq 0$ and analytic in $\operatorname{Re} s>0$ the same will be true of $\hat{U}$. In our case $\hat{f}(s)$ has a pole at $s=i \omega$; hence so will $\hat{U}$ and its residue will be the term in (3.6).

It will again be true that we can find the limit of the integral in (3.5) by replacing $u$ by its limit value $\operatorname{Re}\left(u_{0} e^{i \omega t}\right)$. We compute this integral by using the left side of (3.3). We have, by (I),

$$
\begin{gathered}
\sigma(L, t)=\operatorname{Re} \mathscr{F}\left[U_{x}^{t}(L, 0)\right] \sim \operatorname{Re}\left(\sigma_{0} e^{i \omega t}\right) \\
\sigma_{0}=\varphi_{0} \hat{F}(i \omega) \gamma(i \omega) \operatorname{ctnh} \gamma(i \omega) L
\end{gathered}
$$

and $D \varphi(t) \sim \operatorname{Re} i \omega e^{i \omega t}$. Hence

$$
\begin{aligned}
\int_{t}^{t+2 \pi / \omega} p[u](\tau) d \tau & \sim \frac{1}{2} \operatorname{Re} \int_{t}^{t+2 \pi / \omega}\left(-i \omega \sigma_{0} \bar{\varphi}_{0}\right) d \tau \\
& =\pi \operatorname{Im}\left(\sigma_{0} \bar{\varphi}_{0}\right)=\pi \operatorname{Im}\left(\gamma(i \omega) \operatorname{ctnh} \gamma(i \omega) L \hat{\mathscr{F}}(i \omega)\left|\varphi_{0}\right|^{2}\right) .
\end{aligned}
$$

Now by (3.4) $\mathscr{F}(i \omega) \gamma(i \omega)=i \omega \sqrt{\hat{F}(i \omega)}$ and hence (3.12) yields (II).

We investigate the asymptotic limits of $P(\omega)$ as $\omega \rightarrow \infty$.

For $(\mathrm{K})$ : Here $\hat{\mathscr{F}}(i \omega)=E+\lambda i \omega$. Hence $\operatorname{Im}(i \omega \hat{\mathscr{F}}(i \omega)) \sim \pi(\sqrt{\lambda} \cos \pi / 4) \omega^{3 / 2}$.

For (V): From $(2.15)_{1}$,

$$
\gamma(s)=s(s \hat{a} /(s))^{-1 / 2} \sim \frac{s}{\sqrt{a(0)}}+\delta+O\left(\frac{1}{s}\right), \quad \delta=-\frac{1}{2} \frac{D a(0)}{a(0)^{3 / 2}} .
$$

Thus

Also

$$
\operatorname{ctnh} \gamma(i \omega) L \sim \frac{\sinh 2 \delta L-i \sin (2 \omega L / \sqrt{a(0)})}{\cosh 2 \delta L-\cos (2 \omega L / \sqrt{a(0)})} .
$$

Hence,

$$
i \omega \sqrt{\hat{\mathscr{F}}(i \omega)} \sim \sqrt{a(0)} i \omega .
$$

$$
P(\omega) \sim \pi \sqrt{a(0) \omega} \frac{\sinh 2 \delta L}{\cosh 2 \delta L-\cos (2 \omega L / \sqrt{a(0)})}=O(\omega) .
$$

For $(\mathrm{V}-\mathrm{K})\left(\mathrm{C}_{\mathrm{VK}}\right)$ yields

$$
\gamma(s)=s(\hat{\mathscr{F}}(s))^{-1 / 2} \sim s\left[M+A_{\alpha} s^{\alpha}\right]^{-1 / 2} \sim A_{\alpha}^{-1 / 2} s^{1-\alpha / 2} .
$$

It follows that $\operatorname{ctnh} \gamma(i \omega) L \rightarrow 1$ as $\omega \rightarrow \infty$ and $i \omega \sqrt{\mathscr{F}(i \omega)} \sim A_{\alpha}^{1 / 2}(i \omega)^{1+\alpha / 2}$ hence

$$
P(\omega) \sim \pi A_{\alpha}^{1 / 2} \sin \frac{\pi}{2}\left(1+\frac{\alpha}{2}\right) \omega^{1+\alpha / 2} .
$$

For (R-H) (2.18) yields,

$$
\gamma(s)=s\left\{M+\Gamma(1-\alpha)\left(\eta^{\alpha-1}-(s+\eta)^{\alpha-1}\right\} .\right.
$$

Thus $\gamma(s) \sim p s+q s^{\alpha}+O\left(s^{\alpha-1}\right)$ for some constants $p$ and $q$ so that $\operatorname{ctnh} \gamma(s) L \rightarrow 1$ as $s \rightarrow \infty$. Also

$$
\operatorname{Im} i \omega \hat{\mathscr{F}}(i \omega) \sim\left(M+\Gamma(1-\alpha) \beta^{\alpha-1}\right) \omega .
$$


4. Thermal damping in bars. We consider again the one-dimensional bar with displacement $u(x, t)$ on $(0, L)$. We include thermal effects with temperature $\theta(x, t)$, internal energy $e(x, t)$ and heat flux $q(x, t)$. We assume the bar is at rest initially with initial temperature zero. Both ends are insulated, $x=0$ is clamped and $x=L$ has prescribed displacement $\varphi(t)$.

The balance laws (with unit density) are, with $\varepsilon=u_{x}$

$$
\begin{gathered}
u_{t t}(x, t)=\sigma_{x}(x, t), \\
e_{t}(x, t)=-q_{x}(x, t)+\sigma(x, t) \varepsilon_{t}(x, t),
\end{gathered}
$$

the second being conservation of energy. Let $\eta(x, t)$ be specific entropy and let $\psi(x, t)=e(x, t)-\theta(x, t) \eta(x, t)$ be specific free energy. Put $g(x, t)=\theta_{x}(x, t)$, the temperature gradient. We assume constitutive relations of the form,

$$
\sigma=\hat{\sigma}(\varepsilon, \theta, g), \quad g=-k(\varepsilon, \theta) g, \quad \eta=\hat{\eta}(\varepsilon, \theta, g), \quad \psi=\hat{\psi}(\varepsilon, \theta, g) .
$$

For any process the second law of thermodynamics in the form of the ClausiusDuhem inequality,

$$
\psi_{t}+\theta_{l} \eta+\sigma \varepsilon_{t}+g q(\theta) \leq 0
$$

must hold. It can be shown [4] that this implies the following relations:

$$
k \geq 0, \quad \hat{\psi}_{g}=\hat{\eta}_{g}=0, \quad \hat{\psi}_{\theta}=-\hat{\eta}, \quad \cdot \hat{\psi}_{\varepsilon}=\hat{\sigma} .
$$

We substitute (4.3) and (4.4) into (4.2) and obtain,

$$
\begin{aligned}
0 & =\hat{\psi}_{t}+\theta_{t} \hat{\eta}+\theta \hat{\eta}_{t}-(k g)_{x}-\sigma \varepsilon_{t} \\
& =\hat{\psi}_{\varepsilon} \varepsilon_{t}-\sigma \varepsilon_{t}+\hat{\psi}_{\theta} \theta_{t}+\hat{\eta} \theta_{t}+\theta \hat{\eta}_{t}-(k g)_{x} \\
& =-\theta\left(\hat{\psi}_{\theta}\right)_{t}-(k g)_{x}
\end{aligned}
$$

or

$$
-\theta \hat{\psi}_{\theta \theta} \theta_{t}=\left(k \theta_{x}\right)_{x}+\theta \hat{\psi}_{\varepsilon \theta} \varepsilon_{t} .
$$

We substitute in (4.1) and obtain,

$$
u_{t t}=\hat{\psi}_{\varepsilon \varepsilon} \varepsilon_{x}+\hat{\psi}_{\varepsilon \theta} \theta_{x} .
$$

We now linearize about the equilibrium state $\theta \equiv \theta_{0}$ and $\varepsilon \equiv 0$. This yields,

$$
u_{t l}=E u_{x x}+\alpha \theta_{x}, \quad \theta_{t}=K \theta_{x x}+\beta u_{x t}
$$

where,

$$
\begin{gathered}
E=\hat{\psi}_{\varepsilon \varepsilon}\left(0, \theta_{0}\right), \quad \alpha=\hat{\psi}_{\varepsilon \theta}\left(0, \theta_{0}\right) \\
K=-k / \theta_{0} \hat{\psi}_{\theta \theta}\left(0, \theta_{0}\right), \quad \beta=-\hat{\psi}_{\varepsilon \theta}\left(0, \theta_{0}\right) \theta_{0} \psi_{\theta \theta}\left(0, \theta_{0}\right) .
\end{gathered}
$$

For real materials $\psi_{\theta \theta}<0$ and $\psi_{\varepsilon \varepsilon}>0$ and we will assume $k>0 . \psi_{\varepsilon \theta}$ can be positive or negative but we observe that the constants $\alpha$ and $\beta$ always have the same sign and hence (by scaling) we can assume they are the same so that

$$
u_{t t}=E u_{x x}+\alpha \theta_{x}, \quad \theta_{t}=K \theta_{x x}+\alpha u_{x t} .
$$


We note that if $\alpha=0,(4.8)$ reduces to Hooke's law. Hence whatever damping there is must come from the thermal effects. It is convenient to introduce $v(x, t)=\theta_{x}(x, t)$ so that the problem we solve is:

$$
\begin{gathered}
u_{t t}=E u_{x x}+\alpha v, \quad v_{t}=K v_{x x}+\alpha u_{x x t} \\
u(x, 0)=u_{t}(x, 0)=v(x, 0)=0 \\
u(0, t)=v(0, t)=v(L, t)=0, \quad u(L, t)=\varphi(t) .
\end{gathered}
$$

The power loss calculation of Sec. 3 holds here also with $p[u](t)$ as in (3.3). In this case, however, we must remember that $\sigma(x, t)=E u_{x}(x, t)+\alpha \theta$ where $\theta_{x}=v$.

Our results are the same as in the earlier section $\varphi$ as in (2.3).

THEOREM 4.1. There exists a unique solution $(u, v)$ of (4.9).

THEOREM 4.2. There exists a function $u^{0}(x, \omega)$ such that

$$
u(x, t)=\operatorname{Re}\left(u^{0}(x, \omega) e^{i \omega t}\right)+u^{1}(x, t) \quad u^{1}(x, t) \rightarrow 0 \quad \text { as } t \rightarrow \infty .
$$

TheOREM 4.3. $P(\omega)$ defined by

$$
\int_{t}^{t+2 \pi / \omega} \sigma(L, \tau) D \varphi(\tau) d \tau \rightarrow P(\omega)\left|\varphi_{0}\right|^{2} \quad \text { as } t \rightarrow \infty
$$

exists and $P(\omega)=O(\omega)$ as $\omega \rightarrow \infty$.

The ideas are the same as before but the computations are more complicated. We let $U$ and $V$ be complex solutions for $\varphi(t)=\varphi_{0} e^{i \omega t}+F(t)$. We transform and find

$$
\begin{gathered}
s^{2} \hat{U}=E \hat{U}_{x x}+\alpha V, \quad s \hat{V}=K \hat{V}_{x x}+\alpha s \hat{U}_{x x} \\
\hat{U}(0, s)=\hat{V}(0, s)=\hat{V}(L, s)=0, \quad \hat{U}(L, s)=\hat{f}(s) .
\end{gathered}
$$

We write the equation as

$$
\mathbf{P} \hat{\mathbf{U}}_{x x}=\mathbf{Q} \hat{\mathbf{U}} \quad \text { or } \quad \mathbf{U}_{x x}=\mathbf{A} \hat{\mathbf{U}}
$$

with

so that

$$
\hat{\mathbf{U}}=\left(\begin{array}{c}
\hat{U} \\
\hat{V}
\end{array}\right), \quad \mathbf{P}=\left(\begin{array}{cc}
E & 0 \\
s \alpha & k
\end{array}\right), \quad \mathbf{Q}=\left(\begin{array}{cc}
s^{2} & -\alpha \\
0 & s
\end{array}\right)
$$

$$
\mathbf{A}=\left(\begin{array}{cc}
\frac{s^{2}}{E} & -\frac{\alpha}{E} \\
-\frac{s^{3} \alpha}{K E} & \frac{s \alpha^{2}}{K E}+\frac{s}{K}
\end{array}\right)
$$

We diagonalize. Let $(\lambda(s))^{2}$ and $(\mu(s))^{2}$ be the eigenvalues of $\mathbf{A}$ with eigenvectors,

$$
\begin{aligned}
\mathbf{e}(s)=\left(\begin{array}{c}
-1 \\
e(s)
\end{array}\right), & e(s)=\frac{E}{\alpha}\left(\lambda^{2}-\frac{s^{2}}{E}\right) \\
\mathbf{g}(s)=\left(\begin{array}{c}
-1 \\
e(s)
\end{array}\right), & g(s)=\frac{E}{\alpha}\left(\mu^{2}-\frac{s^{2}}{E}\right) .
\end{aligned}
$$

Now put,

$$
\mathbf{T}(s)=\left(\begin{array}{cc}
-1 & -1 \\
e(s) & g(s)
\end{array}\right), \quad \mathbf{T}^{-1}(s)=\frac{1}{e(s)-g(s)}\left(\begin{array}{cc}
g(s) & 1 \\
-e(s) & -1
\end{array}\right) .
$$


Then if

$$
\left(\begin{array}{c}
\hat{U} \\
\hat{V}
\end{array}\right)=\mathbf{T}(s)\left(\begin{array}{c}
M \\
V
\end{array}\right)
$$

we have,

$$
\begin{gathered}
M_{x x}=\lambda^{2} M, \quad N_{x x}=\mu^{2} N \\
\left(\begin{array}{c}
M(0) \\
N(0)
\end{array}\right)=\left(\begin{array}{l}
0 \\
0
\end{array}\right), \quad\left(\begin{array}{c}
M(L) \\
N(L)
\end{array}\right)=T^{-1}(s)\left(\begin{array}{l}
1 \\
0
\end{array}\right) \hat{\varphi}(s) .
\end{gathered}
$$

We obtain then,

$$
\left(\begin{array}{l}
\hat{U} \\
\hat{V}
\end{array}\right)=T(s)\left(\begin{array}{cc}
\frac{\sinh \lambda x}{\sinh \lambda L} & 0 \\
0 & \frac{\sinh \mu x}{\sinh \mu L}
\end{array}\right) T^{-1}(s)\left(\begin{array}{l}
1 \\
0
\end{array}\right) \hat{\varphi}(s) .
$$

From (4.17) we have,

$$
\begin{aligned}
\left(\begin{array}{l}
\hat{U}_{x}(L, s) \\
\hat{V}_{x}(L, s)
\end{array}\right) & =T(s)\left(\begin{array}{cc}
\lambda \operatorname{ctnh} \lambda L & 0 \\
0 & \mu \operatorname{ctnh} \mu L
\end{array}\right) T^{-1}(s)\left(\begin{array}{l}
1 \\
0
\end{array}\right) \hat{\varphi}(s) \\
& =\frac{1}{e-g}\left(\begin{array}{c}
-\lambda g \operatorname{ctnh} \lambda L+\mu e \operatorname{ctnh} \mu L \\
e g \lambda \operatorname{ctnh} \lambda L-e g \mu \operatorname{ctnh} \mu L
\end{array}\right) \hat{\varphi}(s) .
\end{aligned}
$$

In the appendix we outline a proof that the above calculations are meaningful in the same sense as in the previous section. This means the following. If $\hat{\varphi}(s)$ is continuous in $\operatorname{Re} s \geq 0$ and analytic in $\operatorname{Re} s>0$ then so are $\hat{U}$ and $\hat{V}$. In the present case, however, $\hat{\varphi}(s)$ will have a pole at $s=i \omega$ with residue $f_{0}$. It will follow then that $\hat{U}$ and $\hat{V}$ will have a pole at $s=i \omega$ with residues $u^{0}(x, \omega)$ and $v^{0}(x, \omega)$ given by the right side of (4.17) with $\hat{f}$ replaced by $f_{0}$ and $s$ set equal to $i \omega$. This will yield the relation $(\mathrm{I})$.

We can use (4.18) to obtain the result (II) but we have to be a little careful. Recall that our original energy balance law was $\theta_{t}=k \theta_{x x}+\alpha u_{x t}=k v_{x}+\alpha u_{x t}$. Transforming we obtain $\hat{\theta}=\left(k \hat{v}_{x}\right) / s+\alpha \hat{u}_{x}$. Recall that $\sigma$ is given by $\sigma=E u_{x}+\alpha \theta$ hence we obtain $\hat{\sigma}=\left(E+\alpha^{2}\right) \hat{u}_{x}+\alpha \frac{k}{s} \hat{v}_{x}$ or in the complex from

$$
\hat{\sigma}=\left(E+\alpha^{2}\right) \hat{U}_{x}+\alpha \frac{K}{S} \hat{V}_{x} .
$$

The formula (4.19) and our remarks above show that $\hat{\sigma}(L, s)=\hat{\sigma}_{0}(L, \omega)(s-i \omega)^{-1}+$ regular terms and this corresponds to,

$$
\sigma(L, t)=\sigma_{0}(L, \omega) e^{i \omega t}+\cdots
$$

where the dots indicate terms going to zero as $t \rightarrow \infty$. This is the same situation as in (3.11) and we obtain in the same way

$$
\int_{t}^{t+2 \pi / \omega} p[u](\tau) d \tau \sim \pi \operatorname{Im} \sigma_{0}(L, \omega) \bar{\varphi}_{0} .
$$

We will have, from (4.18) and (4.19)

$$
\sigma_{0}(L, \omega)=\left(E+\alpha^{2}\right) \hat{U}_{x}(L, i \omega)-\frac{\alpha K i}{\omega} \hat{V}_{x}(L, i \omega)
$$


where the terms on the right of (4.22) are computed, as above, by putting $\hat{\varphi}=\varphi_{0}$ and $s=i \omega$ in (4.18). Clearly this will yield (II) but the estimate for $P(\omega)$ requires a very careful computation.

We begin with the observation that $\lambda^{2}(i \omega)$ and $\mu^{2}(i \omega)$ are the roots of the equation

$$
Z^{2}+Z\left(\frac{\omega^{2}}{E}-\frac{i \omega}{K}-\frac{i \alpha^{2} \omega}{K E}\right)-\frac{i \omega^{3}}{K}=0
$$

Hence we have,

$$
\begin{aligned}
2_{\mu^{2}(\omega)}^{\lambda^{2}(\omega)}= & -\frac{\omega^{2}}{E}+\frac{i \omega}{K}+\frac{i \alpha^{2} \omega}{K E} \\
& \pm \sqrt{\frac{\omega^{4}}{E^{2}}+\frac{2 i \omega^{3}}{K E}-\frac{2 i \alpha^{2} \omega^{3}}{K E^{2}}-\frac{\omega^{2}}{K^{2}}-\frac{\alpha^{4} \omega^{2}}{K^{2} E}-\frac{2 \alpha^{2} \omega^{2}}{K^{2} E}} .
\end{aligned}
$$

If we expand for large $\omega$ we find,

$$
\begin{gathered}
\lambda^{2}(\omega)=\frac{i \omega}{K}+O(1) \\
\mu^{2}(\omega)=-\frac{\omega^{2}}{E}+\frac{i \alpha^{2} \omega}{K E}+O(1)
\end{gathered}
$$

so that

$$
\begin{gathered}
\lambda(\omega)=\sqrt{\frac{i \omega}{K}}+O(1), \quad \operatorname{ctnh} \lambda L \rightarrow 1 \\
\mu(\omega)=\frac{i \omega}{\sqrt{E}}+\delta, \quad \delta=\frac{1}{2} \frac{\alpha^{2}}{K \sqrt{E}} \\
\operatorname{ctnh} \mu L \sim \frac{\sinh 2 \delta L-i \sin (2 \omega L / \sqrt{E})}{\cosh 2 \delta L-\cos (2 \omega L / \sqrt{E})} \\
e(\omega)=\frac{E}{\alpha}\left(\frac{i \omega}{K}+\frac{\omega^{2}}{E}\right)+O(1), \quad g(\omega)=\frac{i \alpha \omega}{K}+O(1) \\
e(\omega) \cdot g(\omega)=\frac{\omega^{2}}{\alpha}+O(\omega) .
\end{gathered}
$$

When these formulas are substituted into (4.21) one finds

$$
P(\omega) \sim \pi \omega \sqrt{E} \frac{\sinh 2 \delta L}{\cosh 2 \delta L-\cos (2 \omega L / \sqrt{E})} .
$$

REMARK 4.1. By comparing (4.24) with (3.14) we see that the asymptotic power loss due to thermal damping is of the same form as that for a viscoelastic material. Thus it is possible to specify a viscoelastic material which, for high frequencies, will have the same power loss as that due to thermal damping. From (3.13), (3.14), (4.23), and (4.24) it follows that this equivalence will hold provided $a(0)=E$ and $D a(0)=-\alpha^{2} E / K$. For the original formulation (4.7) and a bar with mass density $\rho$ the expression for $D a(0)$ becomes $D a(0)=-\alpha \beta E / \rho K$. For the prototype viscoelastic kernel this leads to $\eta=\alpha \beta / \rho K$. 


\section{Appendix proofs.}

Proof of Theorem 2.1. We write $\hat{R}(s)=\left(s^{2}+\hat{F}(s)\right)^{-1}$. Then we decompose formula (2.7) as

$$
\begin{gathered}
\hat{\mathscr{E}}(s)=\hat{R}(s)\left\{f_{0}(s-i \omega)+\hat{F}(s)\right\} \\
=\varepsilon_{0}(s-i \omega)^{-1}+\hat{\mathscr{E}}_{1}(s) \\
\varepsilon_{0}=\hat{R}(i \omega) \hat{f}_{0} \\
\mathscr{E}_{1}(s)=\hat{R}(s) \hat{F}(s)+\{\hat{R}(s)-\hat{R}(i \omega)\}(s-i \omega)^{-1} .
\end{gathered}
$$

We substitute (A.1) into (2.8) and obtain

$$
\begin{gathered}
\mathscr{E}(t)=\varepsilon_{0} e^{i \omega t}+\mathscr{E}_{1}(t) \\
\mathscr{E}_{1}(t)=(2 \pi i)^{-1} \int_{c-i \infty}^{c+i \infty} e^{s t} \hat{\mathscr{E}}_{1}(s) d s .
\end{gathered}
$$

LemMA A.1. The integral in (A.4) is independent of $c$ for $c \geq 0$ and defines a function $\mathscr{E}_{1} \in C^{(2)}(0, \infty) \cap C^{(1)}[0, \infty)$ with $\mathscr{E}_{1}(0)=-\varepsilon_{0}, D \mathscr{E}^{1}(0)=-i \omega \varepsilon_{0}$. Moreover $D^{j} \mathscr{E}_{1}(t) \rightarrow$ 0 as $t \rightarrow \infty$ for $j=0,1$.

Proof. Condition (F) and the dissipativity of $\mathscr{F}$ shows that $\hat{\mathscr{E}} \in \mathscr{L}$ except for possible trouble at $s=i \omega$. Our moment conditions on $a, b_{\alpha}$ and $m_{\alpha}$ guarantee that $\hat{\mathscr{F}}(s)$ is differentiable at $s=i \omega$ so that the singularity in $\hat{\mathscr{E}}_{1}$ at $s=i \omega$ is removable. We have to analyze the behavior of $\hat{\mathscr{E}}_{1}(s)$ for large $s$. Note first that by $(\mathrm{F})$, we have, in analogy to (2.15),

$$
\hat{F}(s)=O\left(s^{-2}\right) \text { as } s \rightarrow \infty .
$$

We see from our large $s$ assumptions and (2.15) that in all cases we have $\hat{R}(s)=$ $s^{-2}+O\left(s^{-3}\right)$ for large $s$. Also $(s-i \omega)^{-1}=s^{-1}+i \omega s^{-2}-\omega^{2} s^{-3}+O\left(s^{-4}\right)$. Thus by (A.5) we have

$$
\begin{gathered}
\hat{\mathscr{E}}_{1}(s)=r_{1} s^{-1}+r_{2} s^{-2}+r_{3} s^{-3}+O\left(|s|^{-4}\right), \\
r_{1}=-\hat{R}(i \omega) f_{0}, \quad r_{2}=-i \omega \hat{R}(i \omega) f_{0}, \quad \text { as } s \rightarrow \infty .
\end{gathered}
$$

Let $\varphi_{j}(t)=\left(t^{j-1} e^{-t}\right) /(j !)$ so that $\hat{\varphi}_{j}(s)=(s+1)^{-j}$. Then we can find $z$ such that,

$$
\hat{\mathscr{E}}_{1}(s)=r_{1} \hat{\varphi}_{1}(s)+\left(r_{2}+r_{1}\right) \hat{\varphi}_{2}(s)+z \hat{\varphi}_{3}(s)+\hat{\rho}(s)
$$

where $\hat{\rho}(s)=O\left(|s|^{-4}\right)$. We can write, then,

$$
\begin{gathered}
\mathscr{E}_{1}(t)=r_{1} \varphi_{1}(t)+\left(r_{2}+r_{1}\right) \varphi_{2}(t)+z \varphi_{3}(t)+\rho(t) \\
\rho(t)=(2 \pi)^{-1} \int_{-\infty}^{+\infty} e^{i \eta t} \hat{\rho}(i \eta) d \eta .
\end{gathered}
$$

Since $\hat{\rho}(i \eta)=O\left(\eta^{-4}\right)$ we see that $\rho(t)$ is twice differentiable on $[0, \infty)$ and $D^{j} \rho \in$ $L_{1}(0, \infty)$ for $j=0,1,2$ hence $D^{j} \rho(t) \rightarrow 0$ as $t \rightarrow \infty$ for $j=0,1$. Moreover, the Paley-Wiener theorem gives $\rho(t) \equiv 0$ in $\rho \leq 0$. In particular $D^{j} \rho(0)=0 j=0,1$; hence $\mathscr{E}_{1}(0)=r_{1}=-\hat{R}(i \omega) f_{0}$ and $D \mathscr{E}_{1}(0)=-r_{1}+r_{2}-r_{1}=r_{2}=-i \omega \hat{R}(i \omega)$. But we have $\left.\varepsilon_{0} e^{i \omega t}\right|_{t=0}=\hat{R}(i \omega) f_{0}$ and $\left.D \varepsilon_{0} e^{i \omega t}\right|_{t=0}=-i \omega \hat{R}(i \omega) f_{0}$.

We conclude from Lemma (A.1) that (A.3) defines a twice continuously differentiable function on $t>0$ with $\mathscr{E}(0)=D \mathscr{E}(0)=0$. 
Remark A.1. We extend $\mathscr{E}$ to $t \leq 0$ by making it identically zero. The resulting function will have a discontinuity in its second derivative at $t=0$. If we want to overcome this, we need only require that $F(0)=-R f_{0}$ in $(\mathrm{F})$.

It remains to show that we really have a solution of $(2.2)$. We sketch the argument for $(\mathrm{V}-\mathrm{K})$. We calculate as follows, using $\mathscr{E}(0)=0$ on $t \leq 0$ :

$$
\begin{aligned}
\mathscr{F}\left(\mathscr{E}^{t}\right)= & M \mathscr{E}(t)+\int_{0}^{t} b_{\alpha}(\tau) D \mathscr{E}(t-\tau) d \tau \\
= & (2 \pi i)^{-1}\left\{\int_{c-i \infty}^{c+i \infty} e^{s t \hat{\mathscr{E}}}(s)\left[M+s \int_{0}^{t} b_{\alpha}(\tau) e^{-s \tau} d \tau\right] d s\right\} \\
= & (2 \pi i)^{-1}\left\{\int_{c+i \infty}^{c+i \infty} e^{s t \hat{\mathscr{E}}}(s)\left(M+s \hat{b}_{\alpha}(s)\right) d s\right. \\
& \left.\quad-\int_{c-i \infty}^{c+i \infty}\left[\hat{\mathscr{E}}(s) \int_{t}^{\infty} b_{\alpha}(\tau) e^{s(t-\tau)} d \tau\right] d s\right\} .
\end{aligned}
$$

We claim that the last term in (A.9) is zero. To see this observe that the integrand in the $s$ integral is an analytic function of $s$ in $\operatorname{Re} s>0$ and vanishes as $s \rightarrow \infty$. Hence we can close the contour to the right and conclude that the term is zero. Now $\left(M+s \hat{b}_{\alpha}(s)\right) \hat{\mathscr{E}}(s)=-s^{2} \hat{\mathscr{E}}(s)+\hat{f}(s)$ and $s^{2} \hat{\mathscr{E}}(s)$ is the transform of $D^{2} \mathscr{E}$ so we see that (A.3) satisfies $(\mathrm{V}-\mathrm{K})$. The other models have similar treatments and we have proved Theorem 2.1.

Proof of Theorem 3.1. We have to show that the formula (3.9) is meaningful for $\operatorname{Re} \geq 0$.

Lemma A.2. The quantity $\sinh \gamma(s) L$ does not vanish in $\operatorname{Re} s \geq 0$.

Proof. We show that it cannot vanish on $\operatorname{Re} s=0$ and then it will follow that it cannot vanish in $\operatorname{Re} s \geq 0$. Suppose it is zero for $s=i \omega$. Then the function $\psi(x)=\sinh \gamma(i \omega) x$ solves the problem,

$$
-\omega^{2} \psi=\hat{\mathscr{F}}(i \omega) \psi^{\prime \prime}, \quad 0<x<L, \quad \psi(0)=\psi(L)=0 .
$$

Hence

$$
-\omega^{2} \int_{0}^{L}|\psi|^{2} d x+\hat{\mathscr{F}}(i \omega) \int_{0}^{L}\left|\psi^{\prime}(x)\right|^{2} d x=0 .
$$

Thus we would have to have,

$$
\operatorname{Im} \hat{\mathscr{F}}(i \omega)=0 .
$$

For $(\mathrm{K}) \hat{\mathscr{F}}(i \omega)=E+i \lambda \omega$ and (A.9) can hold only if $\omega=0$ and then (A.8) would give $\psi^{\prime}(x) \equiv 0$ and hence $\psi(x)=0$. For $(\mathrm{V}-\mathrm{K}) \operatorname{Im} \hat{\mathscr{F}}(i \omega)=\operatorname{Im}\left(i \omega \hat{b}_{\alpha}(i \omega)\right)=\omega \operatorname{Re} \hat{b}_{\alpha}(i \omega)$ which is nonzero for $\omega \neq 0$. We have $\hat{\mathscr{F}}(0)=M$ so (A.8) for $\omega=0$ would again imply $\psi \equiv 0$. For $(\mathrm{R}-\mathrm{H}) \operatorname{Im} \hat{\mathscr{F}}(i \omega) \neq 0$ for $\omega \neq 0$ and $\hat{\mathscr{F}}(0)>0$. For $(\mathrm{V})$ we have $\operatorname{Im} \hat{\mathscr{F}}(i \omega)=\operatorname{Im} i \omega \hat{a}(i \omega)=\omega \operatorname{Re}(i \omega) \neq 0$ for $\omega \neq 0$ and $\hat{\mathscr{F}}(0)=a_{\infty}$.

We see then that (3.9) is well defined. We can then argue (pointwise in $x$ ) as we did for the fiber case that the formula (3.10) indeed yields a solution. The details are tedious and essentially as before and thus we omit them. 
Proof of Theorem 4.1. The key again is to make sure that the formula (4.17) is meaningful in $\operatorname{Re} s \geq 0$. It is clear that this formula will be valid unless the homogeneous problem (4.10) has a nontrivial solution. Again we show that this cannot happen.

Lemma A.3. Let $\psi, \chi$ be a solution of (4.10) with $\hat{\varphi}=0$ in $\operatorname{Re} s \geq 0$. Then $\psi \equiv 0$, $\chi \equiv 0$.

Proof. One can again show it suffices to verify the result for $s=i \omega$. Let $\psi, \chi$ be such a solution for some $\omega$. We have then,

$$
\begin{gathered}
-\omega^{2} \int_{0}^{L}|\psi|^{2} d x+E \int_{0}^{L}\left|\psi^{\prime}\right|^{2} d x=\alpha \int_{0}^{L} \chi \bar{\psi} d x \\
i \omega \int_{0}^{L}|\chi|^{2}+K \int_{0}^{L}\left|\chi^{\prime}\right|^{2} d x=-\alpha \int_{0}^{L} \psi^{\prime} \bar{\chi}^{\prime} d x \\
-\omega^{2} \int_{0}^{L} \psi \bar{\chi}+E \int_{0}^{L} \psi^{\prime} \bar{\chi}^{\prime} d x=\alpha \int_{0}^{L}|\chi|^{2} d x \\
i \omega \int_{0}^{L} \chi \bar{\psi} d x+K \int_{0}^{L} \chi^{\prime} \bar{\psi}^{\prime} d x+E \int_{0}^{L}\left|\psi^{\prime}\right|^{2} d x=0 .
\end{gathered}
$$

From these formulas we draw the following conclusions:

$$
\begin{gathered}
\operatorname{Im} \int_{0}^{L} \chi \bar{\psi} d x=-\operatorname{Im} \int_{0}^{L} \psi \bar{\chi} d x=0 \\
\operatorname{Im} \int_{0}^{L} \psi^{\prime} \bar{\chi}^{\prime} d x=-\operatorname{Im} \int_{0}^{L} \bar{\psi}^{\prime} \chi^{\prime} d x=0 \\
\int_{0}^{L}|\chi|^{2} d x=0
\end{gathered}
$$

Hence for $\omega \neq 0 \chi(x) \equiv 0$. But then $\alpha i \omega \psi^{\prime \prime}(x)=0$ with $\psi(0)=\psi(L)=0$; so $\psi(x) \equiv 0$. The case $s=0$ is immediate.

The remainder of the proof of Theorem 4.1 has again the same type of calculations as in the other theorems.

\section{REFERENCES}

[1] C. W. Bert, Material damping, J. Sound and Vibration 29, 129-153 (1973)

[2] M. A. Boit, Linear thermodynamics and the mechanics of solids, Proc. Third U.S. National Congress on Applied Mechanics (1958), pp. 1-18

[3] U. J. Choi and R. C. MacCamy, A fractional order Volterra equation with applications to elasticity, J. Math. Anal. Appl. 132, 448-464 (1989)

[4] B. D. Coleman, Thermodynamics of materials with memory, Archive for Rational Mechanics and Analysis, Vol. 17, (1964), pp. 1-46

[5] W. J. Hrusa and M. Renardy, On wave propagation in linear viscoelasticity, Quart. Appl. Math. 43, 237-253 (1985)

[6] R. C. MacCamy and J. S. W. Wong, Stability theorems for some functional equations, Trans. Amer. Math. Soc. 164, 1-37 (1972)

[7] M. Renardy, Some remarks on the propagation and non-propagation of discontinuities in linearly viscoelastic liquids, Rheological Acta. 21, 251-254 (1982) 